\title{
ON REALISING MOD-2 HOMOLOGY CLASSES OF MANIFOLDS BY SUBMANIFOLDS
}

\author{
by STAVROS PAPASTAVRIDIS
}

(Received 20th September 1977)

\section{Statement of results}

In his fundamental paper (3), Thom proved, among other things, that a mod-2 homology class of an $n$-dimensional, closed, compact, $C^{\infty}$ manifold, which has dimension $\leqslant n / 2$, can be realised by a submanifold, (see (3), Théorème II.1 and Corollaire II.13).

In this note we examine the question of realisability of mod-2 homology classes of the next higher dimension.

Throughout this note "manifold" will mean "closed, compact, $C^{\infty}$ manifold".

From now on, $M$ will be an $(2 n+1)$-dimensional manifold, $z \in H_{n+1}\left(M ; Z_{2}\right)$ will be an $(n+1)$-dimensional mod-2 homology class, and $u \in H^{n}\left(M ; Z_{2}\right)$ its dual cohomology class.

Our main results are the following.

Theorem 1. If $\boldsymbol{n}$ is of the form $2^{r}-1$, then $z$ is always realisable by a submanifold.

Theorem 2. Let $n$ be an odd number not of the form $\left(2^{r}-1\right)$. Then $z$ can be realised by a submanifold, if and only if

(i) $u \cdot \mathrm{Sq}^{1} u+\mathrm{Sq}^{n} \mathrm{Sq}^{1} u=0$, or

(ii) at least one of the Wu classes $v_{n-1}, v_{n-3}, \ldots, v_{n-2^{r}+1}, \ldots$, of $M$, is non-zero.

Remark. The $i$-dimensional $W u$ class of $M$, can be defined by the property $\mathrm{Sq}^{i} x=v_{i} \cdot x$, where $x \in H^{*}\left(M ; Z_{2}\right) v_{i} \in H^{i}\left(M ; Z_{2}\right)$ and $\operatorname{deg}\left(\mathrm{Sq}^{i} x\right)=\operatorname{dim} M$, (see (1), p. 39).

Theorem 3. If $n$ is an odd number not of the form $2^{r}-1$, then there is an $(2 n+1)$-dimensional, $(n-1)$-connected manifold $M$, which has an $(n+1)$-dimensional mod-2 homology class, which cannot be realised by a submanifold.

Theorem 4. If $n$ is an even number and $M$ is orientable, then all mod-2, $(n+1)$-dimensional homology classes of $M$, can be realised by submanifolds.

\section{Proof of Theorems $1,2,3,4$}

Our results depend heavily on P. J. Ledden's paper (2). 
Let $M O(n)$ be the Thom space of the universal $n$-linear bundle. Then, (following Ledden's notation) there a product of $K\left(Z_{2}\right.$, )'s $\bar{K}$ and a map $\bar{F}: M O(n) \rightarrow \bar{K}$ which induces an isomorphism in mod- 2 cohomology, up to dimension $2 n$. Ledden computes the first Postnikov invariant of the map $\bar{F}$, let us call it $\theta$.

Specifically he finds the results stated here as Lemmas 5, 6 and 7.

Lemma 5. If $n=2^{r+1}-1$, then $\theta=0$, (see (2), Lemma 2).

Lemma 6. If $n$ is odd and $2^{r}<n<2^{r+1}-1$, then

$$
\theta=\epsilon_{0} \mathrm{Sq}^{1} \epsilon_{0}+\mathrm{Sq}^{n} \mathrm{Sq}^{1} \epsilon_{0}+\mathrm{Sq}^{n-1} \epsilon_{1}+\mathrm{Sq}^{n-3} \epsilon_{2}+\cdots \mathrm{Sq}^{n-2^{r+1}} \epsilon_{r}
$$

The $\epsilon_{i}$ 's are fundamental classes of factors of $\bar{K}$, such that deg $\epsilon_{i}=2^{r}+n$ if $i \geqslant 1$ and $\epsilon_{0}=$ the Thom class of $M O(n)$. For details on the $\epsilon_{i}$ 's see (2), and particularly Lemma 2 .

Lemma 7. If $n$ is even then $\theta \in H^{2 n+1}(\bar{K} ; Z)$ is a class of finite order, (see (2), Lemma $2^{\prime}$ ).

Lemma 8. The homology class $z$ is realisable by a submanifold if and only if there is a map $f: M \rightarrow \bar{K}$ such that $f^{*}\left(\epsilon_{0}\right)=u$ and $f^{*}(\theta)=0$.

Proof. Obvious by Théorème II.1 of (3) and the fact that $\theta$ is the appropriate Postnikov invariant.

Proof of Theorem 1. Since $\epsilon_{0}$ is the fundamental class of a factor of $\vec{K}$, there is a map $f: M \rightarrow \bar{K}$ such that $f^{*}\left(\epsilon_{0}\right)=u$. Because $\theta=0$ (by Lemma 5) the conditions of Lemma 8 are satisfied, and the result follows.

Proof of Theorem 2. First we prove necessity. Let us assume that the class $z$ can be realised by a submanifold. Then from Lemmas 8 and 6, there is a map $f: M \rightarrow \bar{K}$ such that $u \mathrm{Sq}^{1} u+\mathrm{Sq}^{n} \mathrm{Sq}^{1} u+\mathrm{Sq}^{n-1} f^{*}\left(\epsilon_{1}\right)+\mathrm{Sq}^{n-3} f^{*}\left(\epsilon_{2}\right)+\cdots+\mathrm{Sq}^{n-2^{r+1}} f^{*}\left(\epsilon_{r}\right)=\mathbf{0}$. But this means that either $u \mathrm{Sq}^{1} u+\mathrm{Sq}^{n} \mathrm{Sq}^{1} u=0$, or that at least one of the terms $\mathrm{Sq}^{n-2^{i+1}} f^{*}\left(\epsilon_{i}\right)$ is non zero, for $i=1,2, \ldots, r$. But this last condition implies that one of the $v_{n-2} i_{+1}$ 's of $M$ is non zero.

Proof of sufficiency. The key remark in order to prove sufficiency, is that a map $f: M \rightarrow \bar{K}$ can be defined, such that $f^{*}\left(\epsilon_{0}\right)=u$ and the $f^{*}\left(\epsilon_{1}\right), f^{*}\left(\epsilon_{2}\right), \ldots, f^{*}\left(\epsilon_{r}\right)$ have any preassigned values. That ends the proof.

Proof of Theorem 4. It is exactly the same as Theorem 1. By Lemma 7, for any map $f: M \rightarrow \bar{K}, f^{*}(\theta)=0$ because $H^{2 n+1}(M ; Z)=Z$ and $\theta$ has finite order.

Lemma 9. If $n$ is odd, then there is an $(2 n+1)$-dimensional, $(n-1)$-connected manifold $M$, such that $H_{n}(M ; Z)=Z_{2}$.

Proof. We consider the Stiefel manifold, $V_{n+2,2}=O(n+2) / O(n)$, which is the 
space of all orthonormal 2-frames in $\mathbb{R}^{n+2}$. It is well-known, that $V_{n+2,2}$ is $(n-1)$ connected, and that if $n$ is odd then $\pi_{n}\left(V_{n+2,2}\right)=Z_{2}$. That ends the proof, because $V_{n+2,2}$ is $(2 n+1)$-dimensional.

Lemma 10. Let $n>1$ and let $M$ be an $(n-1)$-connected, $(2 n+1)$-dimensional manifold, such that $H_{n}(M ; Z)=Z_{2}$. Then $H^{n}\left(M ; Z_{2}\right)=Z_{2}$, and if $x$ is the generator of $H^{n}\left(M ; Z_{2}\right)$ then $x \cdot \mathrm{Sq}^{1} x \neq O$.

Proof. By the Universal coefficient theorem and Poincare duality we have:

$$
H^{n}(M ; Z)=H^{n+1}(M ; Z)=O \text { so } H^{n}\left(M ; Z_{2}\right)=Z_{2} \text { and } H^{n+1}\left(M ; Z_{2}\right)=Z_{2} \text {. }
$$

Next we consider the long exact sequence in cohomology of $M$, induced by the short exact sequence $O \rightarrow Z \stackrel{2}{\rightarrow} Z \rightarrow Z_{2} \rightarrow O$. From this we get easily that the Bockstein operator $b_{2}: H^{n}\left(M ; Z_{2}\right) \rightarrow H^{n+1}(M ; Z)$ is an isomorphism and so, by the previous calculations $\mathrm{Sq}^{1}: H^{n}\left(M ; Z_{2}\right) \rightarrow H^{n+1}\left(M ; Z_{2}\right)=Z_{2}$ is an isomorphism. Because of Poincare duality $x \cdot \mathrm{Sq}^{\prime} x \neq O$.

Proof of Theorem 3. Let $M$ be a manifold with the specifications of Lemma 9. Then since it is $(n-1)$-connected we must have $v_{n-1}, v_{n-3}, \ldots, v_{n-2^{r}+1}, \ldots=O$. For the same reason $\mathrm{Sq}^{n} \mathrm{Sq}^{1} x=O$, because $\mathrm{Sq}^{n}$ decomposes. So by the previous Lemma and Theorem 2, the result follows.

\section{REFERENCES}

(1) E. Brown and F. Peterson, Relations among characteristic classes I, Topology 3 (Supplement 1) (1964), 39-52.

(2) P. J. Ledden, Nonstable homotopy groups of Thom complexes, Proc. Amer. Math. Soc. 29 (1971), 404-410.

(3) R. Tном, Quelques propriétès globales des variétés différentiables, Comm. Math. Helv. 28 (1954), 17-86.

UNIVERSITY OF ATHENS

Mathematical Institute

SOLONOS 57

ATHENS 143

GREECE 